\title{
Some experiments on the breakdown effect in apparent motion
}

\author{
DAVID FINLAY \\ University of Newcastle, New South Wales, Australia \\ and \\ MICHAEL VON GRÜNAU \\ Queen's University, Kingston, Ontario, Canada
}

\begin{abstract}
This study was concerned with the breakdown of long-range stroboscopic apparent motion, that is, the cessation of the experience of movement between two sources previously seen in continuous apparent motion. In the first three experiments, the conditions under which breakdown occurs were delimited. The variables employed in these studies were temporal frequency, spatial separation, stimulus duration, interstimulus interval (ISI), stimulus size, and direction of motion. The results indicated that temporal frequency described the data better than did either duration or ISI, that breakdown occurred more readily for greater spatial separations between stimuli, and that within the limits tested, size was not important. Furthermore, continuous motion was more effective than interrupted unidirectional motion in producing breakdown. The final two experiments dealt with the time course of the breakdown for one temporal frequency over a prolonged observation period, and with adaptation effects within the temporal frequency envelope defining the breakdown effects. The limited amount of temporal-frequency-specific adaptation that could be demonstrated suggested that this envelope could best be thought of as describing a single process.
\end{abstract}

When two spatially separated stimuli are presented sequentially under appropriate spatial and temporal conditions, they are reported to move as one object in oscillatory motion. This form of apparent motion (AM) can break down after some time if fixation is maintained near to the path of the AM; that is, the experience will change from that of a single moving point to one of two spatially discrete, flashing points (Kolers, 1964). This breakdown effect is the subject of this paper.

At present, there is only one published paper-that of Anstis, Giaschi, and Cogan $(1985)^{1}$ that describes the conditions that are necessary for the breakdown to occur or for the recurrence of AM that usually follows the breakdown. Interestingly, despite this lack of extensive study, the breakdown effect has been used to support two diametrically opposed viewpoints concerning AM. Kolers (1964) argued that inasmuch as real motion (RM) does not exhibit the breakdown effect, the underlying neural bases for the two motion experiences must be different. In contrast, Clatworthy and Frisby (1973) used the breakdown effect to argue that $\mathrm{AM}$ and $\mathrm{RM}$ are mediated by the same underlying mechanism (i.e., the same neural elements). They measured time to breakdown of AM under a given set of spatiotemporal parameters before and after

This work was completed while the first author was on study leave at Queen's University in 1983. David Finlay's address is Department of Psychology, The University of Newcastle, NSW 2308, Australia. Michael von Grünau's present address is Département de psychologie, Université de Montréal, C. P. 6128, succursale A, Montréal, PQ H3C 3J7, Canada. an adaptation period. Breakdown occurred more quickly when the adaptation was to RM or AM than when stationary or flickering grids were used as adapting stimuli.

The purpose of this paper is not to challenge either of these positions, but rather to provide data that describe the breakdown effect more fully. In addition, the manipulation of variables such as temporal frequency, interstimulus interval, stimulus duration, spatial separation, and stimulus size provides information about possible explanations of the mechanisms that underlie the breakdown effect and, more generally, this type of AM.

In a previous experiment it was demonstrated that the breakdown effect occurs between stereoscopically fused stimuli (Finlay, Manning, Neill, \& Fenelon, in press). The data from this experiment suggested that the breakdown effect occurs centrally and is not determined by retinal events or vergence realignments. Two types of explanation for breakdown as a central effect can be tentatively proposed: an adaptation explanation and an explanation based on a cognitive assessment of the stimuli.

An adaptation explanation of the breakdown effect might propose that somewhere in the visual system exists a class of neurons or neural pathways (Anstis et al., 1985) that are responsive to $A M$ and that become fatigued over time. The neural basis for long-range AM, as opposed to short-range AM, is not established, but the latter is thought to reflect the properties of motion detectors located relatively early in the processing of visual information (Anstis, 1978; Braddick, 1980). An explanation based on a cognitive appraisal of the stimuli might 
propose a changing criterion as the explanation for the breakdown. Throughout the history of AM research, cognitive, or criterion, issues have been consistently raised, and recently Rock (1983) proposed a problem-solving process to account for long-range AM.

The first three experiments of the present study provide essential basic data concerning the breakdown effect, whereas the final two experiments examine the adaptation explanation more directly.

\section{EXPERIMENT 1}

In Experiment 1, measures of the breakdown effect are reported as a function of temporal frequency and spatial separation. Temporal frequency in the case of recycling AM is defined as the number of cycles per second (hertz). One cycle is determined by the sum of the durations of the two stimulus flashes and the two interstimulus intervals (ISIs). Several previous sets of data in the literature describe the probability of occurrence for AM when spatial and temporal parameters (including stimulus on and off times) were varied (e.g., Caelli \& Finlay, 1979; Finlay \& Caelli, 1979; Neuhaus, 1930). It is clear from these data that AM occurred only within a limited range of temporal frequency. Furthermore, this range narrowed as spatial separation was increased. The purpose of the present experiment was to provide equivalent data using time till breakdown (TTB) as a measure, rather than the probability of AM, and to describe the results as a function of temporal frequency.

\section{Method}

Subjects. The subjects were 10 undergraduate students who were naive as to the purpose of the experiment. Their vision was normal or corrected to normal.

Apparatus. Two red $(635 \mathrm{~nm})$ light-emitting diodes (LEDs), $2 \mathrm{~mm}$ in diameter, were used as stimuli. They were separated by either 18 or $37 \mathrm{~mm}$, which at the viewing distance of $54 \mathrm{~cm}$ corresponded to spatial angle separations of $2^{\circ}$ and $4^{\circ}$ of visual angle, respectively. A white screen behind the LEDs provided a homogeneous background.

The LEDs were connected to linked, variable-frequency oscillators, which could be switched to alternate the light sources at one of the following eight temporal frequencies: $0.5,0.75,1.0,2.0$, $3.0,4.0,5.0$, and $6.0 \mathrm{~Hz}$. The stimulus durations were set at $50 \mathrm{msec}$.

Procedure. The subjects were instructed to fixate a point slightly above the midpoint of the AM path and to press a switch when AM was first seen and a second switch if and when the AM ceased. After a full description of AM and familiarizing practice trials, the subjects were presented with 80 stimulus trials. These consisted of 10 repetitions of the eight temporal frequencies and were presented in randomized order. The subjects reported that the task of identifying when breakdown occurred was an easy one (see also Anstis et al., 1985).

The experiment was completed for one spatial separation and then repeated for the other separation. Half of the subjects received the stimuli first at the $2^{\circ}$ separation and then at the $4^{\circ}$ separation; the remaining 5 subjects received the stimuli in the reverse order.

The stimuli were presented until breakdown occurred.

\section{Results}

The obtained data were expressed as mean TTB of the AM experience. TTB clearly varied as a function of temporal frequency for each subject. These data are summarized in Figure 1 as averages for 10 subjects, with spatial separation as the parameter.

An analysis of variance produced the following results: The spatial separation effect $|F(1,18)=4.1, p<.05|$ and the temporal frequency main effect $[F(7,126)=16.7$, $p<.05]$ were significant, whereas the interaction term was not significant.

\section{Discussion}

The results of Experiment 1 indicate that for the stimuli employed here AM was most resistant to breakdown in the region between 2.0 and $3.0 \mathrm{~Hz}$. Furthermore, breakdown was more likely to occur with the larger separation. These data are similar in appearance to those obtained by Finlay and Caelli (1979), who used probability of occurrence of AM. Those data also peaked at around 2.0 to $3.0 \mathrm{~Hz}$.

The results obtained by Anstis et al. (1985) were different in several respects. They used a different measure of the breakdown effect (probability of seeing motion rather than flicker within a fixed exposure time). Probability of seeing motion was a decreasing function of temporal frequency, and no breakdown occurred for $2.0-\mathrm{Hz}$ oscillations. Lower frequencies were not tested, however. Probability of motion also decreased with spatial separation, but the maximum separation tested was $1^{\circ}$ of visual angle. The absence of a breakdown in their data (at least

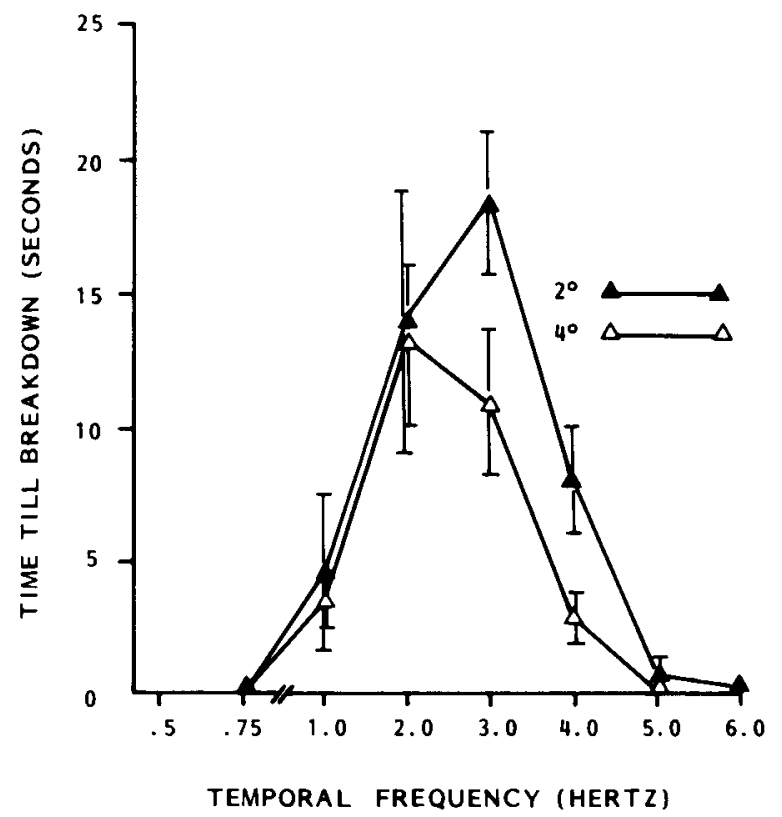

Figure 1. Average time-till-breakdown durations as a function of temporal frequency for two spatial separations. 
within $30 \mathrm{sec}$ ) at $2.0 \mathrm{~Hz}$ might be explained by the smaller separation.

\section{EXPERIMENT 2}

In Experiment 1 the stimulus duration was kept constant, whereas temporal frequency (and, concurrently, ISI) was varied. In Experiment 2, stimulus duration was varied for several levels of temporal frequency by adjusting ISI appropriately. In addition, stimulus size and shape were varied to permit examination of the effects of form on the breakdown of AM.

\section{Method}

Subjects. The 3 subjects were 1 of the authors (D.F.) and 2 practiced but naive observers. All had normal or corrected-to-normal vision.

Apparatus. A three-field tachistoscope (Gerbrands) was used for the stimulus presentation. The stimuli were placed in the first two channels, while the third channel was used to present a continuous fixation point. All stimuli were back-illuminated to avoid the apparent movement of other features within the tachistoscope fields. The stimuli were a pair of round points of light, $2 \mathrm{~mm}$ in diameter, which corresponded to $0.2^{\circ}$ of visual angle; a pair of vertically oriented bars, each $0.2^{\circ} \times 1^{\circ}$; or a pair of vertically oriented bars, each $0.2^{\circ} \times 2^{\circ}$.

The five temporal frequencies used were 1.0, 1.5, 2.0, 4.0, and $5.0 \mathrm{~Hz}$. Within each of these frequency values, stimulus durations were set at $5,25,50$, or $100 \mathrm{msec}$ by varying the ISI values. To achieve some degree of comparability with the previous experiment, a spatial separation of $2^{\circ}$ was used throughout.

Procedure. The stimuli were presented in blocks of stimulus size (the points of light, the small bars, and the large bars). The order of presentation was varied for the 3 subjects. Within these blocks, the subjects received 200 trials consisting of 10 repetitions of the 20 combinations of temporal frequency and stimulus duration.
Within a given trial the stimulus duration was the same for both stimuli.

As in Experiment 1, the subject had to press a switch when AM was first seen and press a second switch if and when AM ceased.

\section{Results}

An analysis of variance of the TTB data indicated no effect for either stimulus size $[F(2,4)=0.29, p>.05]$ or stimulus duration $[F(3,6)=1.48, p>.05]$, but a strong effect for temporal frequency $[F(4,8)=5.96$, $p<.01]$, similar to that found in Experment 1. Although not significant, the data for the duration $\times$ frequency interaction are shown in Figure 2. The curves, in descending order, are $2.0 \mathrm{~Hz}, 1.5 \mathrm{~Hz}, 4.0 \mathrm{~Hz}, 1.0 \mathrm{~Hz}$, and $5.0 \mathrm{~Hz}$, indicating little or no apparent motion at the lowest and highest temporal frequency and most AM around $2.0 \mathrm{~Hz}$, as was reported in Experiment 1. The data are shown because they are at a variance with those reported by Anstis et al. (1985), who obtained an effect of duration. It is clear from these data that the stimulus duration magnitude is not an important variable when compared with temporal frequency (or stimulus onset asynchrony, SOA). This issue was taken up again in Experiment 3 .

\section{EXPERIMENT 3}

Many studies based on single-cell recording within the visual system have shown the existence of directionally specific motion-selective cells (e.g., Barlow \& Levick, 1965; Van Essen \& Maunsell, 1983). Likewise, psychophysical studies have shown the existence of direction selective mechanisms in the human visual system, (e.g.,

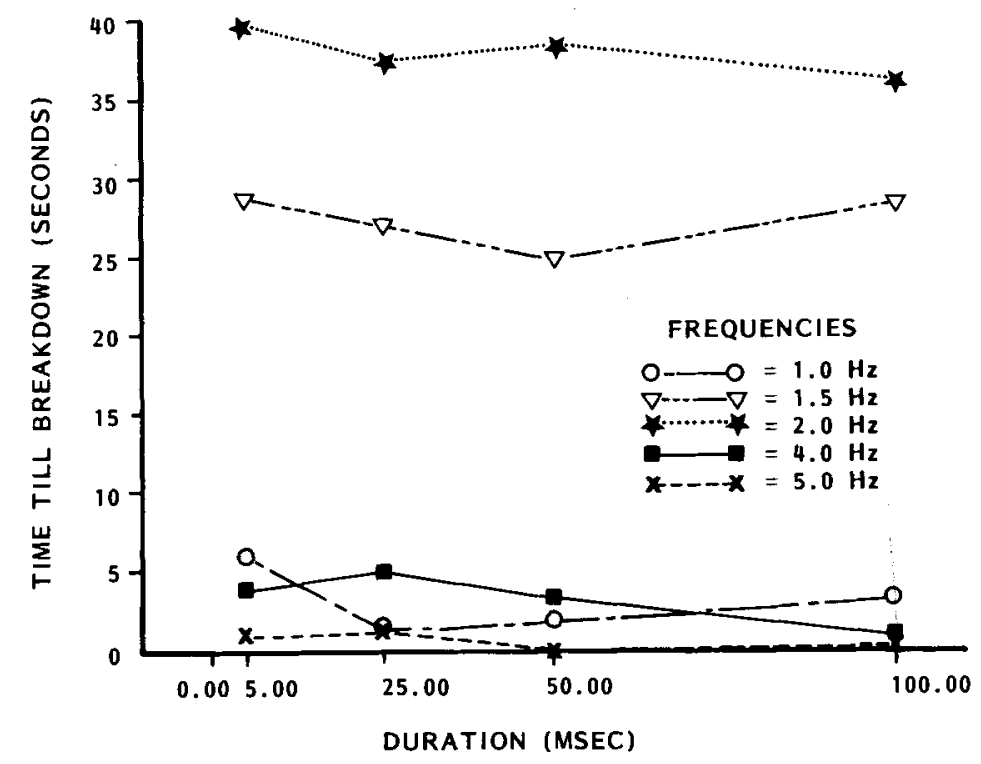

Figure 2. Average time-till-breakdown values for the duration $\times$ frequency interaction term in Experiment 2. The four duration values $(5,25,50$, and $100 \mathrm{msec})$ are shown on the abscissa and the various frequencies are shown in the legend. 
Levinson \& Sekuler, 1976). Furthermore, these mechanisms have been shown to be weakened by adaptation (motion aftereffect). Recently, it has been reported that the motion aftereffect also exists for long-range AM (von Grünau, 1986).

Condition 1 in Experiment 3 consisted of continuous bidirectional movement, as in Experiments 1 and 2. When optimal motion was seen, this display appeared to consist of smooth back-and-forth oscillation of a stimulus bar.

Conditions 2 and 3 presented motion in one direction only, either to the right or to the left, with a sufficiently large time interval (a cycle break time, or CBT) between the end of the second stimulus and the reappearance of the first stimulus to preclude the appearance of motion in the opposite direction. These displays appeared as smooth motion of a bar in one direction, followed by a pause and then by motion in the same direction, and so on.

Condition 4 consisted of bidirectional motion, but the cycling was interrupted by the CBT after each back-andforth oscillation. The sequence of stimulus presentations was as follows: Stimulus 1, ISI, Stimulus 2, ISI, Stimulus 1 , CBT, Stimulus 2, and so on. In this display, a bar appeared to move smoothly first in one direction and then the other, repeating the movements after a short pause.

In this experiment, four different cycling conditons were used in order to examine the influence of motion direction. For all conditions, spatial separation was kept constant, and within each cycling condition both ISI and duration were varied.

\section{Method}

Subjects. Four subjects were used: 2 were experienced in making judgments about AM; 2 were inexperienced and were naive as to the purpose of this experiment. All had normal or corrected-tonormal vision.

Stimuli. The stimuli were two vertically oriented rectangles, $0.2^{\circ} \times 0.85^{\circ}$, separated by a distance of $1^{\circ}$ of visual angle at a viewing distance of $57 \mathrm{~cm}$. A Gerbrands four-field tachistoscope was used for stimulus presentation and timing control.

Procedure. Each subject was presented with each of the four cycling conditions. Within each of these conditions, three stimulus durations $(5,25$, and $50 \mathrm{msec})$ and three values for ISI $(50,75$, and $100 \mathrm{msec}$ ) were used. The CBT for Conditions 2, 3, and 4 was $400 \mathrm{msec}$.

The subjects were required to indicate if and when motion appeared after the start of the trial sequence and if and when the motion ceased. Each subject made five judgments for each combination of the levels of the three variables (condition, duration, and ISI). Each trial sequence was limited to $25 \mathrm{sec}$. As in the other experiments, a fixation point was provided above the path of AM.

\section{Results}

The following analysis is based on the average amount of time for which apparent motion was seen for each of the $4 \times 3 \times 3$ stimulus conditions by each of the 4 subjects. These data were analyzed by a four-way repeated measures analysis of variance (Trials $\times$ ISI $\times$ duration $\times$ cycling condition).

Because of the use of the CBT, temporal frequency is not an appropriate comparison measure for the various cycling conditions, and the discussion will initially be in terms of ISI and duration.

The analysis of variance resulted in the following significant main effects: ISI $[F(2,6)=147.37, p<.01]$, duration $[F(2,6)=135.76, p<.01]$, and cycling condition $[F(3,9)=6.89, p<.01]$. The following interaction terms were also significant: ISI $\times$ duration $[F(4,12)$ $=24.00, p<.01]$, ISI $\times$ condition $[F(6,18)=5.78$, $p<.01]$, duration $\times$ condition $[F(16,18)=3.86$, $p<.02]$, and ISI $\times$ duration $\times$ condition $[F(12,36)=$ $3.86, p<.01]$. These data are summarized in Figure 3, which shows the ISI $\times$ duration $\times$ condition interaction.

According to these data, breakdown occurred most readily for the continuous cycling condition and showed little difference among the other three conditions. Furthermore, it appears that breakdown occurred more readily for shorter ISIs or stimulus durations. When the data for Condition 1 (continuous cycling) were replotted in terms of temporal frequency instead of ISI (see Figure 4), the effect of duration was no longer present and, as in Experiments 1 and 2, the probability of breakdown simply increased as the upper temporal limits of AM were reached. This was true also of the other three conditions, but there was some difficulty in defining temporal frequency because of the use of the CBT.

\section{EXPERIMENT 4}

Experiments 1-3 delimited a number of variables important in describing the occurrence of the breakdown effect; however, they said little directly about the processes that might underlie the effect. Two types of explanation were introduced earlier; one based on adaptation and the other based on a problem-solving process. The adaptation model would predict a certain amount of regularity in the time course of the breakdown process. In the problem-solving model, the experience of AM is considered to be "'a solution to the problem posed by the rather unusual stimulus sequence" (Rock, 1983, p. 166). There are several difficulties in applying this viewpoint to the breakdown effect. First, with the formation of a solution to a particular set of spatial and temporal conditions, it is not clear why the experience of motion should suddenly cease. For a problem-solving model to be relevant, it would need to explain why a given set of spatial and temporal conditions give rise, successively, to motion and nonmotion experiences. There is nothing in the problemsolving approach that leads to a prediction about the timing of such criterion shifts, other than that they should perhaps be unpredictable and should vary widely across subjects.

In Experiment 4, the time course of the breakdown effect was studied over a long period for one temporal frequency $(2.0 \mathrm{~Hz})$. Use was made of the observation that over a period of time AM breaks down, recurs, breaks down, and so on. The prediction would be that if an adaptation process were in operation, the time course of break- 

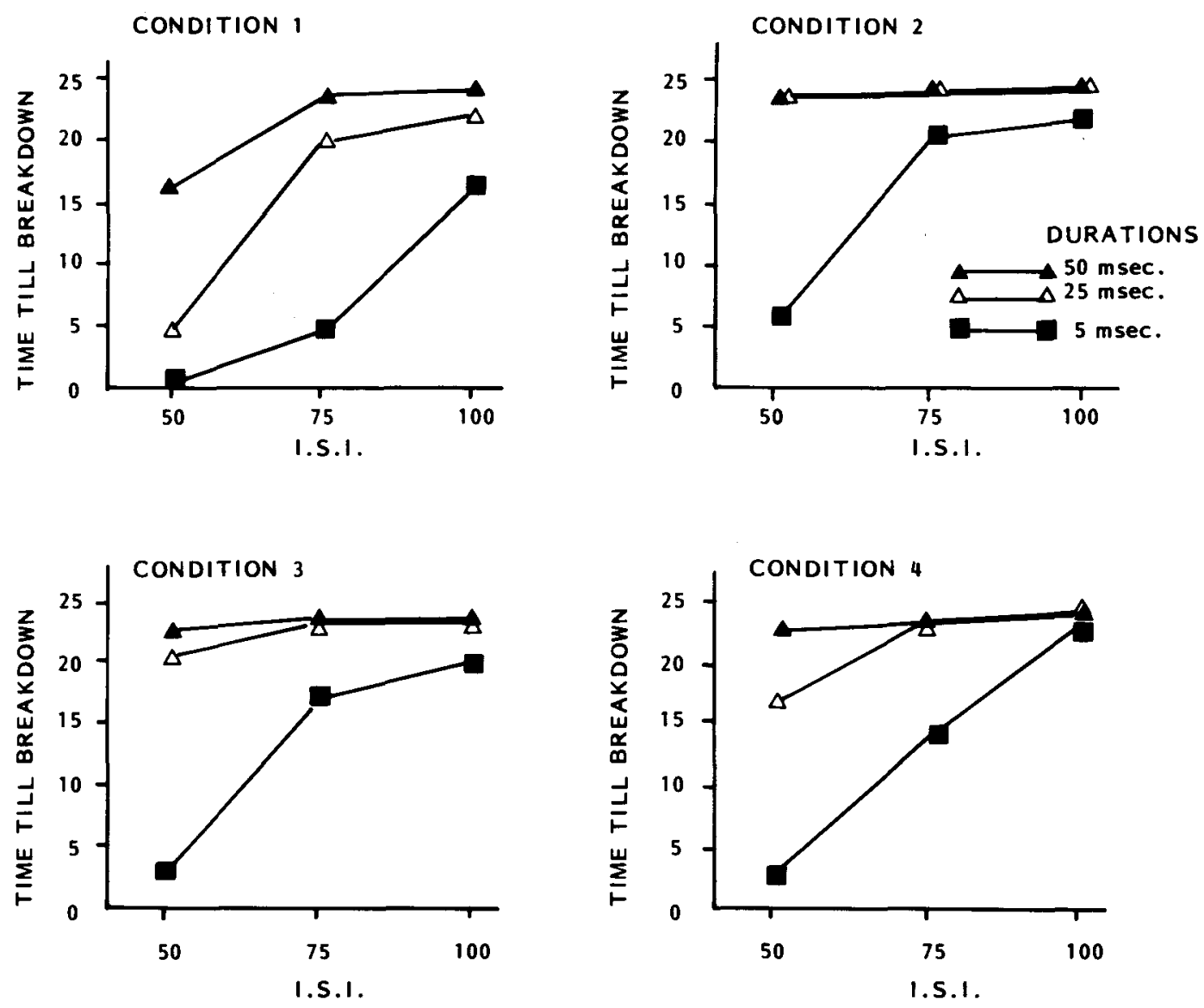

Figure 3. Average time till breakdown as a function of interstimulus interval (ISI) for each of the four cycling conditions. The parameter for the three separate curves is stimulus duration.

down and recurrence of motion would be a regular one. The probability of breakdown would be expected to increase with increased viewing time, leading to a decrease over time for the duration of motion episodes. The cognitive view would not predict such regularity, and any criterion change would be expected to be independent of viewing time.

\section{Method}

Subjects. Five subjects were used-3 experienced in making AM judgments and 2 inexperienced. Except for 1 of the experienced viewers, all were naive as to the purpose of the experiment. All had normal or corrected-to-normal vision.

Stimuli. The stimuli were the same as those used in Experiment 3 but with a spatial separation of $2^{\circ}$ of visual angle. The stimulus duration was $25 \mathrm{msec}$ and only one continuous cycling rate was used, namely $2 \mathrm{~Hz}$. Each subject completed 20 trials and each trial was programmed to last for $2.5 \mathrm{~min}$, during which time the subject was required to fixate a red point equidistant between the two stimulus elements.

The procedure was as follows: When ready, the subject initiated a trial. He/she responded by pushing a button when motion first occurred, when it broke down, when (if) it started up again, and so on. This was done for the duration of the stimulus exposure period. The times were recorded by computer and consisted of the duration of the first motion period, the duration of the first motion breakdown period, the duration of the second motion period, and so on. This was continued until the trial ended.

\section{Results}

The data were variable, both across subjects and across trials, with respect to the number of motion periods and nonmotion periods that were reported. Average motion and nonmotion periods were calculated for the first eight reversals between motion and nonmotion, but the number of data points entering into each mean diminished with the number of reversals because of this variability. For instance, the number of data points for the first motion period was 20 for all subjects, but was reduced to 7 for some subjects for the fourth motion period and to 3 for the fourth nonmotion period. To avoid ceiling effects due to the fixed duration of the trial, motion or nonmotion periods were used only if followed by further periods of motion or nonmotion.

These data, averaged over subjects, are shown in Figure 5 together with the standard errors of the mean.

Both motion and nonmotion periods decreased systematically over the trial period in a nearly parallel fashion, with nonmotion periods always briefer than motion periods. The relative uniformity across subjects is indi- 


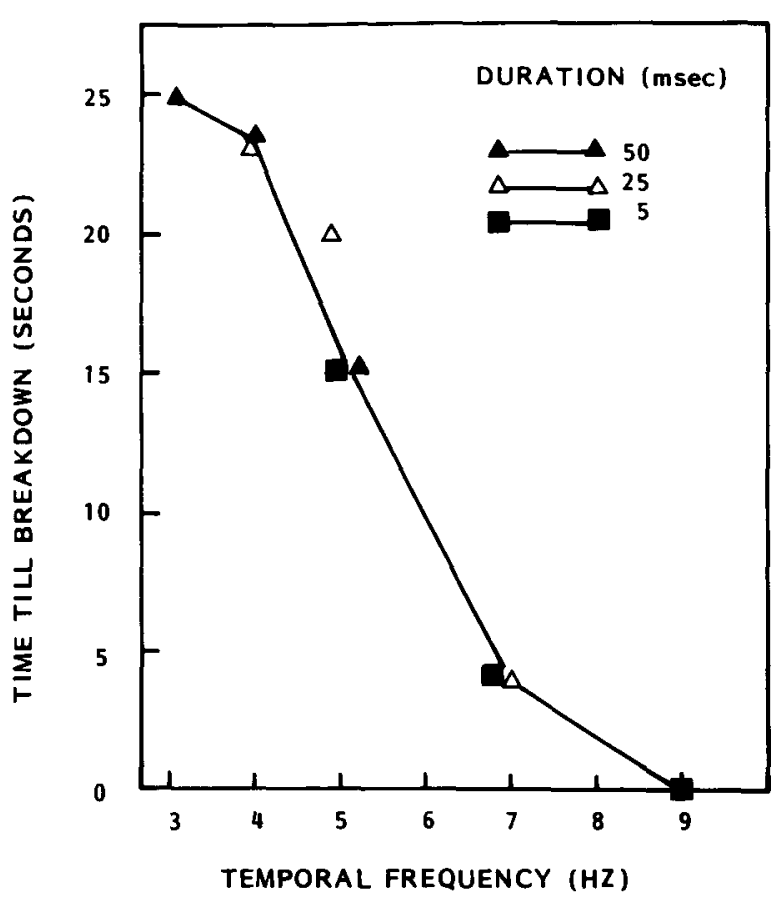

Figure 4. Replotting of the data for Condition 1 shown in Figure 3. This figure shows the time till breakdown as a function of temporal frequency with the parameter of stimulus duration.

cated by the size of the standard errors. These results suggest that the breakdown effect is an indication of the operation of an adaptation process.

\section{EXPERIMENT 5}

The results reported for the first four experiments suggest that the breakdown measure provided some indication of the stability of AM. Experiment 4 furthermore suggested the operation of an adaptation process. The object of Experiment 5 was to see if this stability was affected by prior prolonged viewing of pairs of lights presented at different temporal frequencies. That is, if breakdown is basically the result of adaptation, prior adaptation of the neural channels involved should affect the breakdown process. As mentioned earlier, Clatworthy and Frisby (1973) showed that TTB was, indeed, influenced by prior viewing of AM. Of specific concern in this experiment, however, was the effectiveness of specific temporal frequencies as adapting stimuli. It is clear from Experiments 1-3 that there is a limited temporal frequency region over which TTB (and occurrence of AM) can be measured. The question posed here is whether, within this range, each temporal frequency is an equally effective adapting stimulus, or whether adaptation is temporalfrequency-specific.

\section{Method}

Subjects. Four subjects were used-2 were experienced in making AM judgments and 2 were inexperienced. One of the ex- perienced viewers and both of the inexperienced viewers were naive as to the purpose of the experiment.

Stimuli. The stimuli were the same as those used in Experiment 3, with the spatial separation between the stimuli set at $2.5^{\circ}$ of visual angle. All stimuli were presented at durations of $25 \mathrm{msec}$ and the ISI was identical for both directions of cycling motion. As in Experiments 1 and 2, this gave the appearance of oscillating motion. Five temporal frequencies $(1.0,1.5,2.0,3.0$, and $4.0 \mathrm{~Hz})$ and 10 adapting stimuli were used. The 10 adapting stimuli also had temporal frequencies of $1.0,1.5,2.0,3.0$, or $4.0 \mathrm{~Hz}$. Since the efficiency of the adapting stimuli might depend on the amount of time for which they were exposed or on the number of motion sequences, the stimuli were equated in two ways. Five of the 10 adapting stimuli were equated for number of oscillations (set at 150 for each temporal frequency) and 5 were equated in terms of exposure time (set at $2.5 \mathrm{~min}$ ).

Procedure. Each subject completed 12 series of tests-2 series without adapting stimuli and 10 with adapting stimuli. For the 2 series without adapting stimuli, each subject was presented with 50 test trials comprising 10 repetitions of the five temporal frequencies. One series was presented at the beginning of the experiment and the second at the end, as a control for practice effects. The subject's task was, as in previous experiments, to indicate when AM began and also when it broke down. When breakdown occurred the trial ceased.

Of the 10 adaptation series one of the $10(2 \times 5)$ adapting stimuli was chosen randomly. The subject was presented with 70 trials50 test trials made up of 10 repetitions of the five temporal frequencies and 20 trials of adapting stimuli randomly interspersed with the $\mathbf{5 0}$ test trials, with the constraint that the first trial was always an adaptation trial. Instead of having one adaptation trial for each test trial, we made the assumption that adaptation effects, if any, would carry across all test trials until the next adaptation trial, which would "top up" the adaptation effect. On those trials classed as adaptation trials, a red light appeared before the trial began and the subject merely had to maintain fixation throughout the period.

For the next adaptation series, another adapting stimulus was selected to be used throughout that testing session.

The entire testing procedure was under computer control, but subjects were able to initiate each trial when ready. The period between successive trials was approximately $3 \mathrm{sec}$. The experiment was run over 12 consecutive days with approximately $1.5 \mathrm{~h}$ of testing per subject per day. Time of day for the testing was controlled for each subject over the 12 days, and the order of the experimental conditions was randomized across subjects and across days, with the constraint mentioned above for the nonadaptation series.

\section{Results}

The data for the nonadaptation series were the TTB values for each test-trial temporal frequency averaged over trials and series (i.e., 20 values per mean per subject). The data for the adaptation trials were the means across the test trials for each temporal frequency.

To investigate whether an adaptation effect occurred, the mean TTB values for each temporal frequency for the nonadaptation trials were subtracted from the corresponding means for the adaptation trials. These change scores are plotted in Figure 6; the fact that nearly all scores are negative indicates that adaptation occurred.

\section{GENERAL DISCUSSION}

The results of our experiments indicated that temporal frequency provided a parsimonious description of the time 


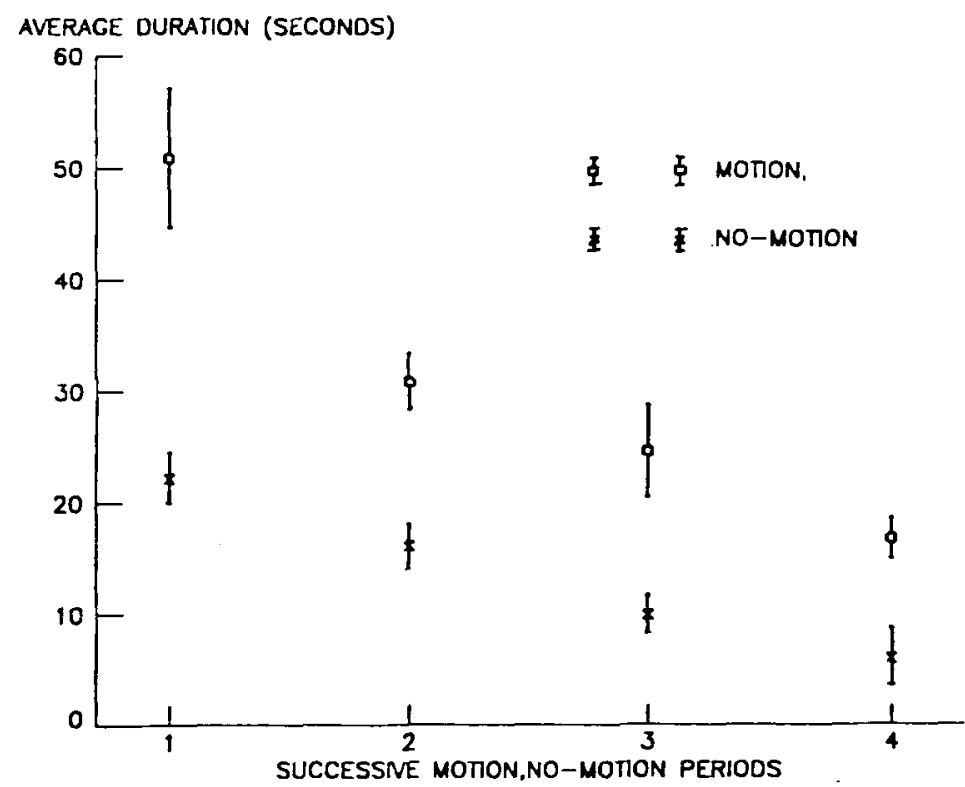

Figure 5. Average durations (in seconds) for four successive motion, no-motion periods. The motion, no-motion periods are indicated in the legend.

parameter for the breakdown of apparent motion. Using this measure it could be demonstrated that TTB occurred within a limited range, or envelope, of temporal frequencies ranging from approximately 1.0 to $5.0 \mathrm{~Hz}$ for the stimuli used here. This range varied with the spatial separation between the light sources but did not vary with stimulus size within the limits tested.

There are indications that TTB also varied with ISI and stimulus duration (see, e.g., Experiment 3), but these data became more manageable when they were converted to the temporal-frequency measure. When the temporalfrequency measure was used, it was clear, especially in Experiments 1-3, that the breakdown occurred more readily on the flanks of the temporal frequency envelopeespecially at the higher frequencies.

These data are confirmed by Experiment 2 (see Figure 2), which showed that whatever the frequency, duration values did not affect TTB values; that is, when ISI and duration were varied but frequency (and thus SOA) was kept constant, TTB stayed constant. These data are at variance with those reported by Anstis et al. (1985), who compared two values of duration within each SOA. In the first value the stimulus duration was combined with a zero ISI and in the second the duration was halved and an equivalent ISI was introduced. Anstis et al. found that their measure of breakdown was more resistant, at every frequency, when the ISI was present. The only apparent resolution between the two sets of data must rest upon further research, but tentatively, it might be suggested that the inclusion of a zero ISI is a special instance that produces different effects.

The shape of the envelope, the behavior of the breakdown effect with size and separation, and a description in terms of temporal frequency rather than duration or ISI measures all mirror the findings for other measures of AM, such as probability of occurrence (Caelli \& Finlay, 1979). This is true whether these other measures were derived from continuously oscillating stimuli or from oneshot stimuli in which the two stimuli were shown only once on each trial. Anstis et al. (1985) used a probabilityof-occurrence measure that was derived from measurements of the breakdown effect (time of seeing motion vs. flicker). Since this measure included episodes of breakdown as well as of recurrence of AM, it cannot be regarded as a direct measure of the breakdown effect, and is thus not comparable to our measure of TTB. Given the parallel between these two kinds of measure of AM, it can be argued that both can be regarded as reflecting a single aspect of AM, namely, its stability or strength. In these terms, probability of occurrence measures directly reflect probability of breakdown.

In other experiments, it has been shown that the breakdown effect occurs dichoptically (Finlay et al., in press). This characteristic of the long-range mechanism differentiates it from the short-range mechanism, which does not occur dichoptically (Braddick 1980; but see Shadlen \& Carney, 1985). Whereas the short-range mechanism may be a reflection of directionally selective neurons (Grusser \& Grusser-Cornehls, 1973), it is not clear what kinds of processes may underlie the long-range mechanism (Braddick, 1980; Larsen, Farrell, \& Bundesen, 1983).

Experiment 3 considered the directionality of the stimuli and its effect on breakdown in more detail. Continuously oscillating AM clearly led to faster breakdown than did unidirectional AM or an oscillating AM with a pause between each oscillation. When the unidirectional motions and the interrupted oscillatory motion were compared, no differences were evident. The results indicate that the continuity of the stimulation, rather than any directionality in the movement, was the important variable. This leads 
to the following explanation: If continuous oscillation leads to a buildup of adaptation, which in turn leads to the breakdown, it could be argued that the interposing of the CBT would allow any accumulated adaptation to at least partially dissipate, masking any possible effects of directionality. This point needs to be the focus of further experimentation. If the difference between these interrupted conditions and the continuous condition can be attributed to the CBT, as pointed out above, the CBT may be used to provide insight into the recovery dynamics of the process.

The idea that the breakdown effect might be the result of a criterion change by the subject needs to be entertained. The results of all the experiments for all subjects were remarkably consistent in overall shape, and all peaked at approximately the same frequency. This implies that the probability of breakdown is strongly tied to temporal frequency and, within a given frequency, is
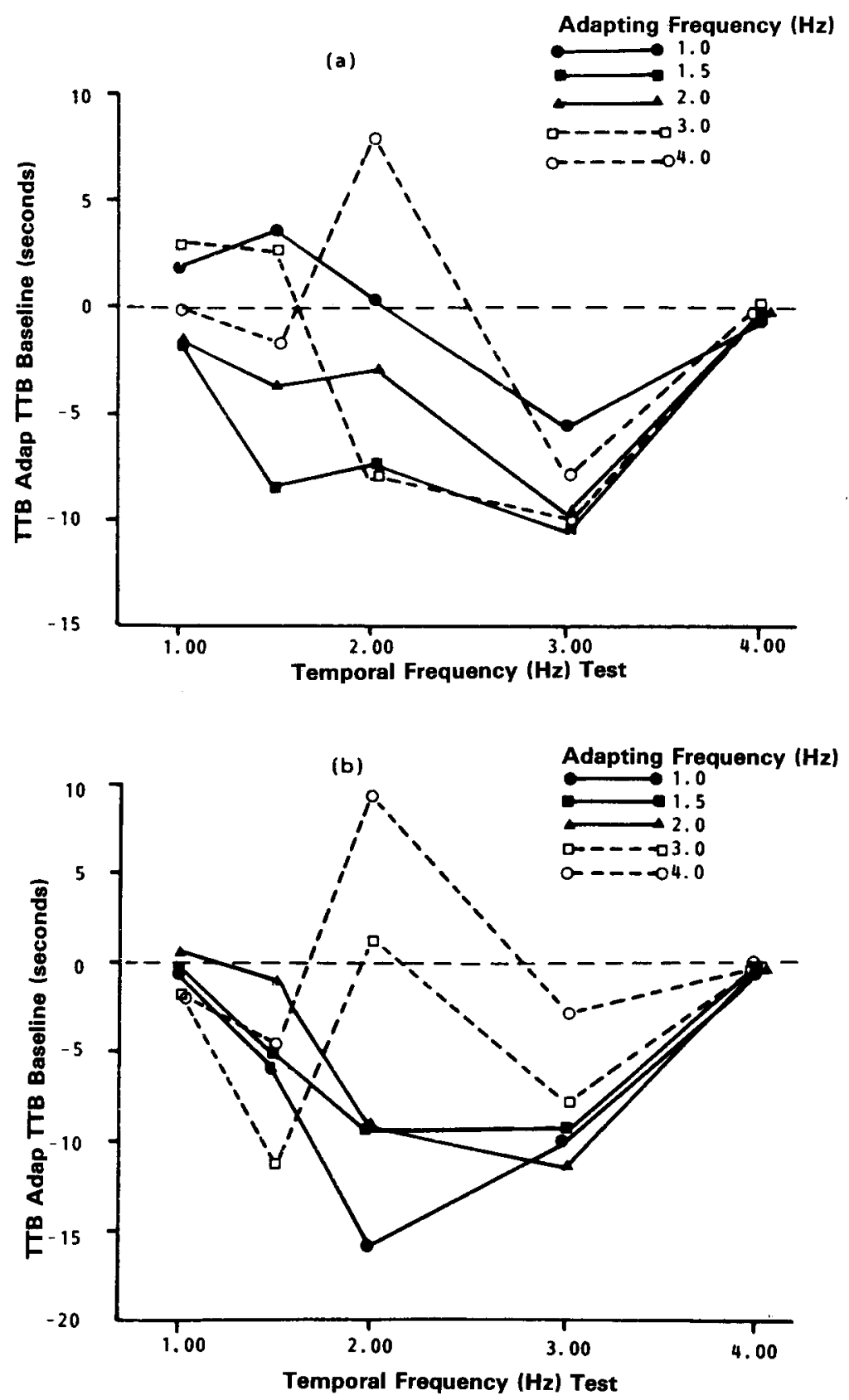

Figure 6. Average time till breakdown (TTB) for adaptation trials minus the TTB for the nonadaptation trials (or baseline) for each temporal frequency of the test stimuli. The adapting stimuli are shown in the legend. (a) Adapting stimuli were equated for the number of oscillations. (b) Adapting stimuli were equated for duration. 
dependent on the exposure time rather than on cognitive events. The curves generated in Experiment 5 further support this view and suggest the operation of an adaptation process rather than one involving a change in criterion.

The final experiment examined the adaptation idea more directly. Compared with the TTB data collected in the nonadapting condition, the TTB data from the lower adapting frequencies $(1.0,1.5$, and $2.0 \mathrm{~Hz})$ were more effective in producing further breakdown than were the $3.0-$ or $4.0-\mathrm{Hz}$ adapting stimuli. This finding is consistent with those of Anstis et al. (1985). It is known (Clatworthy \& Frisby, 1973) that frequencies within this region are also susceptible to adaptation by RM, as well as AM (von Grünau, 1986), but not by flicker or stationary bars (Anstis et al., 1985; Clatworthy \& Frisby, 1973). During the adaptation period, subjects experienced both motion and flicker (due to breakdown of motion). The proportion of flicker and AM within an adaptation period is, of course, related to the adapting frequency, and the higher frequencies may have had less of an adaptation effect because more flicker and less motion occurred with these stimuli. This is a testable idea and needs to be followed up.

In general, our adaptation results are in agreement with the corresponding results of Anstis et al. (1985). Higher temporal frequencies showed greater adaptability, and higher adapting frequencies were less efficient adaptors. Together, these results suggest that AM stimuli with higher temporal frequencies constitute weaker motion signals than do lower-frequency stimuli.

Our results and those of Anstis et al. (1985) àlso agree in that no frequency-specific adaptation effects were found. In contrast, Kruse, Stadler, and Wehner (1986) recently reported direction as well as temporalfrequency-specific effects for the processing of long-range AM. Their task, however, was tied to the adaptation of directional mechanisms, which do not seem to be involved in our task (see Experiment 3).

The adaptation effect over all conditions in the present study was comparatively small, however, which was probably due to the adaptation method used. Others have shown aftereffects to long-range AM stimuli (Anstis \& Moulden, 1970; Banks \& Kane, 1972), but the effects were small and short-lived, especially when compared with adaptation to RM (von Grünau, 1986). The present data can similarly be explained as being due to an adaptation process that varies with temporal frequency and that has a very fast recovery period. The data displayed in Figure 5 show that even under conditions of continuous stimulation, recovery from adaptation occurs, on average, in 20 sec or less.

In conclusion, a limited temporal-frequency range similar to that found for other AM measures nicely describes the breakdown effect. Within this envelope of temporal frequency, breakdown is much more likely to occur at the flanks, especially the higher-temporal-frequency flank. This is true when adapting stimuli are used and when spa- tial separation between the stimuli is increased. Breakdown appears to occur because of an adaptation process, but a process that has a very fast recovery time.

\section{REFERENCES}

ANstis, S. M. (1978). Apparent movement. In R. Held, H. W. Leibowitz, \& H. L. Teuber (Eds.), Handbook of sensory physiology Vol. 8. Berlin: Springer-Verlag.

ANSTIS, S., GIASCHI, D., \& CogaN, A. I. (1985). Adaptation to apparent motion. Vision Research, 25, 1051-1062.

ANStIs, S. M., \& Moulden, B. P. (1970). After effect of seen movement: Evidence for peripheral and central components. Quarterly Journal of Experimental Psychology, 22, 222-229.

Banks, W. P., \& Kane, D. A. (1972). Discontinuity of seen motion reduces the visual motion aftereffect. Perception \& Psychopyshics, 12, 69-72.

BaRLow, H. B., \& Levick, W. R. (1965). The mechanism of directionally selective units in rabbit's retina. Journal of Physiology (London), 178, 477-508.

BRADDICK, $O$. (1980). Low level and high level processes in apparent motion. Philosophical Transactions of the Royal Society of London, B 290, 137-151.

CAELU, T. M., \& Finlay, D. C. (1979). Frequency, phase, and colour coding in apparent motion. Perception, 8, 59-68.

Clatworthy, J. L., \& Frisby, I. P. (1973). Real and apparent movement: Evidence for a unitary mechanism. Perception, 2, 161-164.

FinLAY, D. C., \& CaElli, T. M. (1979). Frequency, phase, and colour coding in apparent motion: II. Perception, 8, 595-602.

Finlay, D., Manning, M., Neill, R., \& Fenelon, B. (in press). Effects of movement in the background field on long-range apparent motion. Vision Research.

GRüSSER, O. J., \& GRüsSER-CORNEHLS, V. (1973). Neuronal mechanisms of visual movement perception and some psychophysical and behavioural correlations. In R. Jung (Ed.), Handbook of sensory physiology: Vol. 7, Pt. 3A. Central processing of vision information. Berlin: Springer-Verlag.

KOLERS, P. A. (1964). Ilusion of movement. Scientific American, 211, 98-108.

Kruse, P., Stadler, M., \& Wehner, T. (1986). Direction and frequency specific processing in the perception of long-range apparent movement. Vision Research, 26, 327-336.

Larsen, A., Farrell, J. E., \& Bundesen, C. (1983). Short- and longrange processes in visual apparent movement. Psychological Research, 45, 11-18.

LEVINSON, E., \& SEKULER, R. (1976). Adaptation alters perceived direction of motion. Vision Research, 16, 779-781.

Neuhaus, W. (1930). Experimentelle Untersuchung der Scheinbewegung. Pflügers Archiv fir die Gesamte Psychologie des Menschen und der Tiere, 75, 315-458.

Rock, I. (1983). The logic of perception. Cambridge, MA: MIT Press.

Shadlen, M., \& CARNEY, R. (1985). Cyclopean perception of motion without depth cues. Investigative Ophthalmology \& Visual Science, 26, 55.

Van Essen, D. C., Maunsell, J. H. R. (1983). Hierarchical organization and functional streams in the visual cortex. Trends in Neurosciences, $6,370-375$.

von GrüNaU, M. W. (1986). A motion aftereffect for long-range stroboscopic apparent motion. Perception \& Psychophysics, 40, 31-38.

\section{NOTE}

1. This paper came to our notice while the present manuscript was being revised.

(Manuscript received June 18, 1985; revision accepted for publication May 22,1987 .) 